\title{
STRESS AND ANXIETY AMONG HEALTHCARE WORKERS ASSOCIATED WITH COVID-19 PANDEMIC IN RUSSIA
}

\author{
Ekaterina Mosolova ${ }^{1}$, Seockhoon Chung ${ }^{2}$, Dmitryi $\operatorname{Sosin}^{3}$ \& Sergey Mosolov ${ }^{3,4}$ \\ ${ }^{I}$ Faculty of Basic Medicine, Lomonosov Moscow State University, Moscow, Russia \\ ${ }^{2}$ Department of Psychiatry, Asan Medical Center, University of Ulsan College of Medicine, Seoul, Korea \\ ${ }^{3}$ Department of Psychiatry, Russian Medical Academy of Continuous Professional Education, \\ Ministry of Public Health of Russian Federation, Moscow, Russia \\ ${ }^{4}$ Moscow Research Institute of Psychiatry, Moscow, Russia
}

received: 23.5.2020;

revised: 20.6.2020;

accepted: 25.9.2020

\section{SUMMARY}

Background: Mental health of medical workers treating patients with COVID-19 is an issue of increasing concern worldwide. The available data on stress and anxiety symptoms among healthcare workers during the COVID-19 are relatively limited and have not been evaluated in Russia yet.

Subjects and methods: The cross-sectional anonymous survey included 1,090 healthcare workers. Stress and anxiety symptoms were assessed using Stress and Anxiety to Viral Epidemics - 9 (SAVE-9) and Generalized Anxiety Disorder - 7 (GAD-7) scales. Logistic regression, Kaiser-Meyer-Olkin two component factor model, Cronbach's alpha and ROC-analysis were performed to determine the influence of different variables, internal structure and consistency, sensitivity and specificity of SAVE-9 compared with GAD-7.

Results: The median scores on the GAD-7 and SAVE-9 were 5 and 14, respectively. 535 (49.1\%) respondents had moderate and $239(21.9 \%)$ had severe anxiety according to SAVE-9. 134 participants (12.3\%) had severe anxiety, 144 (13.2\%) had moderate according to GAD-7. The component model revealed two-factor structure of SAVE-9: "anxiety and somatic concern" and "social stress". Female gender $(O R-0.98, p=0.04)$ and younger age $(O R-0.65, p=0.04)$ were associated with higher level of anxiety according to regression model. The total score of SAVE-9 with a high degree of confidence predicted the GAD-7 value in comparative ROC analysis.

Conclusions: Healthcare workers in Russia reported high rates of stress and anxiety. The Russian version of the SAVE-9 displayed a good ratio of sensitivity to specificity compared with GAD-7 and can be recommended as a screening instrument for detection of stress and anxiety in healthcare workers.

Key words: stress - anxiety - SAVE-9 - GAD-7 - COVID-19 - healthcare workers

$$
* * * * *
$$

\section{INTRODUCTION}

At the close of 2019, the World Health Organization (WHO) was informed of a pneumonia of unknown cause detected in the city of Wuhan in Hubei province, China (Sohrabi et al. 2020). On March 11, 2020, the Director-General of WHO characterized the situation as a pandemic of coronavirus disease 2019 (COVID-19) (World Health Organization, 2020). First COVID-19 cases were diagnosed in Russia on 31 January 2020. By the end of March, many hospitals cancelled all scheduled procedures and started receiving patients with COVID-19. A large group of healthcare workers was involved in the treatment of patients with the novel virus. Studies of previous epidemics have shown high levels of anxiety and stress symptoms in front-line healthcare professionals (Koh et al. 2005, Lui et al. 2012, Hall et al. 2008, Lung et al. 2009). Similarly, the mental health of medical workers treating patients with COVID-19 is now becoming an issue of increasing concern. The first studies from China and Italy report that the current pandemic is no exception (Rossi et al. 2020, Lai et al. 2020).
Several researches also reported of the main factors that contribute to mental health outcomes in medical workers during the pandemic: separation from families, the risk of getting infected, predictable shortages of supplies, an increasing number of COVID-19 cases, speculations about the transmission, lack of definitive treatment protocols (Cai et al. 2020, Ho et al. 2020), extensive media coverage and lack of social support (Mohindra et al. 2020, Xiao et al. 2020).

However, the available data on stress and anxiety symptoms and risk factors associated with them among healthcare workers during the COVID-19 are still relatively limited and have not been evaluated in Russia yet.

Moreover, stress and anxiety during the pandemic may present itself with quiet specific features centering on the risks of infection and major life changes such as separation from families. Thus, it is necessary to develop more precise diagnostic criteria that can be implemented as effective screening instrument during the viral outbreak.

In current study we aimed to evaluate stress and anxiety symptoms among healthcare workers directly involved in the diagnosis and treatment of patients with 
COVID-19 and to analyze potential risk factors that contribute to them during the outbreak in Russia. Another objective was to validate the Russian version of new SAVE-9 scale (Chung et al. 2020). The results obtained in our study may be useful for the development of mental health support programs for healthcare workers during and after the pandemic in order to reduce actual and delayed negative psychiatric consequences.

\section{SUBJECTS AND METHODS}

\section{Subjects}

The study is a cross-sectional hospital-based survey. Data were collected between May $19^{\text {th }}$ and May $26^{\text {th }} 2020$ using an on-line questionnaire spread throughout social networks. The survey was anonymous, and confidentiality of information was assured. The study and the form of the survey were approved by Local Ethical Committee of Moscow Research Institute of Psychiatry, and written informed consent for participation was waived. Most participants worked in the hospitals receiving patients with COVID-19 in Moscow. According to Russian public health organizations, during this period, the number of confirmed COVID-19 cases exceeded 300,000 in Russia and 150,000 - in Moscow.

\section{Methods}

The questionnaire investigated stress and anxiety symptoms. These were assessed using the Russian version of the new SAVE-9 scale (Chung et al. 2020) and the Russian version of GAD-7 scale (Spitzer et al. 2006). We also collected age, gender, occupation and the period of actual work with patients diagnosed with COVID-19. The total score of anxiety using GAD-7 was interpreted as: normal (0-4), mild (5-9), moderate (1014), and severe (15-21) anxiety (Spitzer et al. 2006, Löwe et al. 2008).

The Russian version of SAVE-9 has not been validated and has no official cut-off score available yet. The scale values were split into 3 categories depending on the score as (minor (0-10), moderate (11-19) and severe (20-35)) according to percentiles. The scale includes 9 questions on anxiety (questions 1, 2, 3, 4, 5, and 8) and work-related stress (questions 6, 7, and 9) associated with epidemic (Chung et al. 2020). For the translation in Russian the authors used the English version of SAVE-9. When the first draft was approved by the philologist, back translation was done by the independent experienced psychologist and was verified by the native speaker.

\section{Statistical analysis}

Data analysis was performed using SPSS statistical software version 21.0 (IBM Corp., Armonk, NY). The significance level for demographic characteristics and scale scores was set as $p<0.05$, and for the multiple comparisons two-tailed level of $p<0.015$ has been chosen. The demographic characteristics and scale scores were summarized as mean \pm standard deviation. As all data were not normally distributed according to Kolmogorov-Smirnov test $(p<0.05)$, they were as well presented as medians with interquartile ranges (IQRs). Spearman rank correlation was used to measure the degree of association SAVE-9 and GAD-7 total score.

A multivariable logistic regression model was used in order to explore the association between selected anxiety levels according to GAD-7 and SAVE-9 total scores and age, gender, occupation and the duration of work with patients with COVID-19.

A principal components analysis with Varimax rotation was conducted to clarify the SAVE-9 scale structure and the role of individual items. Internal consistency of the SAVE-9 was evaluated through Cronbach's alpha.

A Receiver Operating Characteristics (ROC) analysis was used to determine the sensitivity and specificity of SAVE-9 for anxiety symptoms evaluation compared with GAD-7. The analyzed variable was the total SAVE-9 score; the GAD-7 group was selected as the status variable.

Table 1. Demographic characteristics of subjects $(\mathrm{N}=1.090)$

\begin{tabular}{lc}
\hline Healthcare workers & \\
Physicians & $468(42.9 \%)$ \\
Intensive care physicians & $80(7.3 \%)$ \\
Nurses & $389(35.7 \%)$ \\
Junior nurses & $90(8.3 \%)$ \\
Paramedics & $55(5 \%)$ \\
Others* & $8(0.7 \%)$ \\
Gender & \\
Female & $740(67.9 \%)$ \\
Male & $350(32.1 \%)$ \\
Age & \\
$\leq 19$ & $10(0.9 \%)$ \\
$20-29$ & $441(40.5 \%)$ \\
$30-39$ & $256(23.5 \%)$ \\
$40-49$ & $226(20.7 \%)$ \\
$50-59$ & $137(12.6 \%)$ \\
$\geq 60$ & $20(1.8 \%)$ \\
Duration & $35.01 \pm 11.22$
\end{tabular}

Duration of work with COVID-19 (days) $35.01 \pm 11.22$

Symptoms assessments

Generalized Anxiety Disorder-7 (GAD-7) $\quad 6.34 \pm 5.75$

Normal $503(46.1 \%)$

Mild $\quad 309(28.4 \%)$

Moderate $144(13.2 \%)$

Severe $134(12.3 \%)$

Stress and Anxiety to Viral $\quad 14.47 \pm 6.58$

Epidemics-9 (SAVE-9)

Minor

$316(29 \%)$

Moderate

535 (49.1\%)

Severe

$239(21.9 \%)$

* laboratory and radiology technicians, administrators 


\section{RESULTS}

The study included 1.090 healthcare workers: 548 physicians $(50.2 \%)$ and 542 nurses and other paramedical personnel (49.8\%) (468 physicians (42.9\%), 80 intensive care physicians (7.3\%), 389 nurses (35.7\%), 90 junior nurses $(8.3 \%), 55$ paramedics $(5 \%)$ and others $(0.7 \%)$ - laboratory and radiology technicians, administrators). Majority of the participants were women - 740 $(67.9 \%)$. The mean age was $35.01 \pm 11.22$ years; the median (IQR) - 33 (19). The mean period of actual work with patients diagnosed with COVID-19 was 34.07 \pm 21.31 days; the median (IQR) - 30 (31).

The mean total scores on the GAD-7 and SAVE-9 were $6.34 \pm 5.75$ and $14.47 \pm 6.58$; the median (IQR) - 5 (9) and 14 (9), respectively. 134 participants (12.3\%) had severe anxiety, $144(13.2 \%)$ - moderate, 309 $(28.4 \%)$ - mild, $503(46.1 \%)$ - normal according to GAD-7 total score interpretation. $316(29 \%)$ had minor, $535(49.1 \%)$ - moderate, and $239(21.9 \%)$ - severe anxiety according to selected SAVE-9 total score interpretation by percentiles.

Demographic characteristics of the participants are presented in Table 1.

The SAVE-9 scores significantly correlated with GAD-7 score $($ rho $=0.59, \mathrm{p}<0.001)$.

The frequencies of participants' answers on each SAVE-9 scale question are presented in Table 2. 707 $(64.9 \%)$ participants often or always have been worried about their family or friends getting infected because of them, $387(35.5 \%)$ - have been more sensitive towards minor physical symptoms, $336(30.8 \%)$ - have been worried about getting infected themselves, 319 (29.3\%) have thought that their colleagues would have more work to do due to their absence from a possible quarantine and might blame them, 235 (21.5\%) - have been afraid their health will worsen because of COVID$19,235(21.5 \%)$ - have been worried about others might avoiding them even after the infection risk has been minimized, 212 (19.4\%) - have been feeling skeptical about their job after going through this experience. Lower number of participants have been worried about the treatment of patients with viral illnesses after this experience and about virus outbreak indefinite continue - $741(68.0 \%)$ and $444(40.7 \%)$ of them have never been concerned about that.

The frequencies of participants' answers on each GAD-7 scale question are presented in Table 3. More than half of the days or almost every day for the last two weeks 347 (31.9\%) participants have been feeling nervous, anxious, or on edge, 344 (31.5\%) have had trouble relaxing, $274(25.1 \%)$ have been easily annoyed or irritable, 261 (24\%) participants have been worrying too much in general, $170(15.6 \%)$ have been unable to control the anxiety, $160(14.7 \%)$ have been feeling afraid as if something awful might happen and 145 $(13.3 \%)$ have been so restless that it's hard to sit still.

The regression SAVE-9 model was reliable $(-2 \mathrm{Log}$ likelihood ratio $=1935.2 ; p=0.05)$. The minor group was used as the reference category. Older age (Odds ratio (OR) 0.980 (95\% confidence interval (CI) 0.965-0.996, $p=0.01)$ ) and male sex (OR 0.655 (95\%CI 0.442-0.971, $p=0.04)$ ) were associated with lower anxiety level among the participants with severe anxiety (see Table 4).

Table 2. The frequencies of participants' answers on each SAVE-9 scale items

\begin{tabular}{|c|c|c|c|c|c|}
\hline & Never & Rarely & Sometimes & Often & Always \\
\hline \multicolumn{6}{|c|}{ Are you afraid the virus outbreak will continue indefinitely? } \\
\hline Total. No. $(\%)$ & $444(40.7)$ & $232(21.3)$ & $301(27.6)$ & $79(7.2)$ & $34(3.1)$ \\
\hline \multicolumn{6}{|c|}{ Are you afraid your health will worsen because of the virus? } \\
\hline Total. No. $(\%)$ & $180(16.5)$ & $263(24.1)$ & $412(37.8)$ & $154(14.1)$ & $81(7.4)$ \\
\hline \multicolumn{6}{|c|}{ Are you worried that you might get infected? } \\
\hline Total. No. (\%) & $133(12.2)$ & $264(24.2)$ & $357(32.8)$ & $217(19.9)$ & $119(10.9)$ \\
\hline \multicolumn{6}{|c|}{ Are you more sensitive towards minor physical symptoms than usual? } \\
\hline Total. No. (\%) & $139(12.8)$ & $249(22.8)$ & $315(28.9)$ & $250(22.9)$ & $137(12.6)$ \\
\hline
\end{tabular}

Are you worried that others might avoid you even after the infection risk has been minimized?

$\begin{array}{llllll}\text { Total. No. (\%) } & 414(38.0) & 198(18.2) & 243(22.3) & 158(14.5) & 77(7.1)\end{array}$

Do you feel skeptical about your job after going through this experience?

$\begin{array}{llllll}\text { Total. No. (\%) } & 471(43.2) & 172(15.8) & 235(21.6) & 140(12.8) & 72(6.6)\end{array}$

After this experience, do you think you will avoid treating patients with viral illnesses?

$\begin{array}{llllll}\text { Total. No. (\%) } & 741(68.0) & 159(14.6) & 107(9.8) & 54(5.0) & 29(2.7)\end{array}$

Do you worry your family or friends may become infected because of you?

$\begin{array}{llllll}\text { Total. No. }(\%) & 57(5.2) & 95(8.7) & 231(21.2) & 320(29.4) & 387(35.5)\end{array}$

Do you think that your colleagues would have more work to do due to your absence from a possible quarantine and might blame you?

Total. No. (\%)

$337(30.9)$

$185(17.0)$

249 (22.8)

$174(16.0)$

$145(13.3)$ 
Table 3. The frequencies of participants' answers on each GAD-7 scale items

Not at all Several days More than half the days Nearly every day

How often have you been bothered by feeling nervous, anxious, or on edge over the past 2 weeks?
Total. No. (\%)
$335(30.7)$
408 (37.4)
$131(12.1)$
$216(19.8)$

How often have you been bothered by not being able to stop or control worrying over the past 2 weeks?

Total. No. (\%)

608 (55.8)

$312(28.6)$

$83(7.6)$

$87(8)$

How often have you been bothered by worrying too much about different things over the past 2 weeks?
Total. No. (\%)
407 (37.3)
422 (38.7)
130 (11.9)

$131(12.1)$

How often have you been bothered by trouble relaxing over the past 2 weeks?
Total. No. (\%)
405 (37.2)
$341(31.3)$
$154(14.1)$
$190(17.4)$

How often have you been bothered by being so restless that it's hard to sit still over the past 2 weeks?
Total. No. (\%)
657 (60.3)
288 (26.4)
82 (7.5)
$63(5.8)$

How often have you been bothered by becoming easily annoyed or irritable over the past 2 weeks?
Total. No. (\%)
$398(36.5)$
418 (38.4)
128 (11.7)

146 (13.4)

How often have you been bothered by feeling afraid as if something awful might happen over the past 2 weeks?

\begin{tabular}{lllll} 
Total. No. $(\%)$ & $579(53.1)$ & $351(32.2)$ & $66(6.1)$ & $94(8.6)$ \\
\hline
\end{tabular}

Table 4. Influence of gender, age, position, time to work with patients with COVID-19 on SAVE-9 scale

\begin{tabular}{lcccc}
\hline Categories & $\mathrm{p}$ & OR & Lower limit & Upper limit \\
\hline Moderate & & & \\
Male & 0.45 & 0.885 & 0.645 & 1.214 \\
Female & 0 & 0 & 0 & 0 \\
Age & 0.17 & 0.991 & 0.979 & 1.004 \\
Time to work with COVID-19 & 0.32 & 1.003 & 0.997 & 1.010 \\
Physicians & 0.11 & 1.274 & 0.945 & 1.718 \\
Nurses & 0 & 0 & 0 & 0 \\
Severe & & & 0.442 & 0.971 \\
Male & 0.04 & 0.655 & 0 & 0 \\
Female & 0 & 0 & 0.965 & 0.996 \\
Age & 0.01 & 0.980 & 0.697 & 1.426 \\
Physicians & 0.99 & 0.997 & 0 & 0 \\
Nurses & 0 & 0 & 0.995 & 1.012 \\
Time to work with COVID-19 & 0.43 & 1.003 & & \\
\hline
\end{tabular}

Table 5. Influence of gender, age, position, time to work with patients with COVID-19 on GAD-7 scale

\begin{tabular}{|c|c|c|c|c|}
\hline Categories & $\mathrm{p}$ & OR & Lower limit & Upper limit \\
\hline \multicolumn{5}{|l|}{ Mild } \\
\hline Male & 0.01 & 0.518 & 0.373 & 0.719 \\
\hline Female & 0 & 0 & 0 & 0 \\
\hline Age & 0.01 & 0.975 & 0.962 & 0.988 \\
\hline Time to work with COVID-19 & 0.92 & 1.000 & 0.993 & 1.007 \\
\hline Physicians & 0.01 & 2.005 & 1.471 & 2.732 \\
\hline Nurses & 0 & 0 & 0 & 0 \\
\hline \multicolumn{5}{|l|}{ Moderate } \\
\hline Male & 0.01 & 0.467 & 0.303 & 0.720 \\
\hline Female & 0 & 0 & 0 & 0 \\
\hline Age & 0.01 & 0.963 & 0.945 & 0.981 \\
\hline Time to work with COVID-19 & 0.30 & 1.005 & 0.996 & 1.014 \\
\hline Physicians & 0.01 & 1.897 & 1.270 & 2.835 \\
\hline Nurses & 0 & 0 & 0 & 0 \\
\hline \multicolumn{5}{|l|}{ Severe } \\
\hline Male & 0.01 & 0.246 & 0.146 & 0.415 \\
\hline Female & 0 & 0 & 0 & 0 \\
\hline Age & 0.01 & 0.950 & 0.931 & 0.969 \\
\hline Time to work with COVID-19 & 0.15 & 1.007 & 0.998 & 1.016 \\
\hline Physicians & 0.46 & 1.172 & 0.773 & 1.778 \\
\hline Nurses & 0 & 0 & 0 & 0 \\
\hline
\end{tabular}


Table 6. Principal components analysis SAVE-9 $(n=1,090)$

\section{Questions}

Factor 1

2. Are you afraid your health will worsen because of the virus?

3. Are you worried that you might get infected?

4. Are you more sensitive towards minor physical symptoms than usual?

8. Do you worry your family or friends may become infected because of you?

Factor 2

1. Are you afraid the virus outbreak will continue indefinitely?

5. Are you worried that others might avoid you even after the infection risk has been minimized?

6. Do you feel skeptical about your job after going through this experience?

7. After this experience. do you think you will avoid treating patients with viral illnesses?

9. Do you think that your colleagues would have more work to do due to your absence from a possible quarantine and might blame you?

Mean \pm SD $\quad$ Factor $1 \quad$ Factor 2

$\begin{array}{lll}1.72 \pm 1.123 & 0.799 & 0.273 \\ 1.93 \pm 1.167 & 0.851 & 0.148 \\ 2.00 \pm 1.213 & 0.749 & 0.146 \\ 2.81 \pm 1.163 & 0.642 & 0.243\end{array}$

$1.11 \pm 1.115$

0.378

0.570

$1.34 \pm 1.304 \quad 0.276$

0.570

$1.24 \pm 1.304$

$-0.41$

0.766

$0.60 \pm 1.027$

0.223

0.524

$1.64 \pm 1.403$

0.162

0.521

Never - 0, Rarely - 1, Sometimes - 2, Often - 3, Always - 4

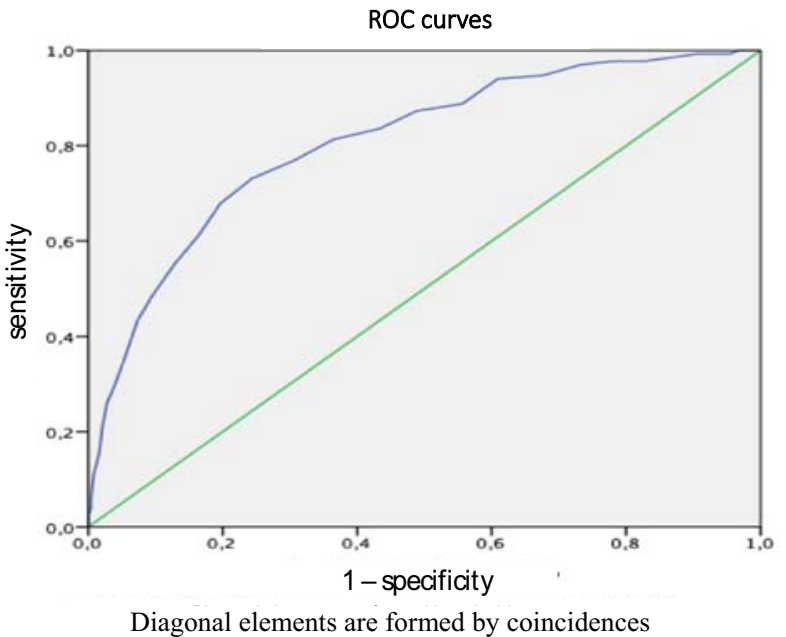

Figure 1. ROC curve sensitivity and specificity of SAVE-9 compared with GAD-7

The regression model for GAD-7 was also reliable $(-2 \log$ likelihood ratio $=2272.5 ; p=0.01)$. The minor group was used as the reference category. Being a physician was associated with more severe anxiety symptoms in participants with mild $(O R=2.005 ; 95 \% \mathrm{CI}$ $1.471-2.732 ; p=0.01)$; and moderate anxiety $(O R=1.897$; $95 \%$ CI 1.270-2.835; $p=0.01)$. Older age was associated with higher anxiety level according to GAD-7 score in participants with mild (OR 0.975 ; 95\% CI 0.962-0.988; $p=0.01)$, moderate (OR 0.963; 95\%CI 0.945-0.981, $p=0.01)$ and severe $(O R=0.95 ; 95 \%$ CI $0.931-0.969$, $p=0.01)$ anxiety. The female gender was a risk factor for a relatively higher GAD-7 score: in mild group $O R=0.518(95 \%$ CI $0.373-0.719, p=0.01)$, in moderate group - OR $0.467(95 \% \mathrm{CI} \quad 0.303-0.720, p=0.01)$, in severe group $-O R=0.246(95 \%$ CI $0.146-0.415, p=0.01)$ (see Table 5).

The two-component model (Kaiser-Meyer-Olkin adequacy criterion $=0.847$, Barletts' sphericity criterion, $p<0.0001$ ) was obtained as a result of the main components analysis describing $51 \%$ of the system. Factor 1 ("anxiety about the viral epidemic") included questions 2,3,4,8 (eigenvalue $=3,497$ ), factor 2 ("work-related stress associated with the viral epidemic") - 1,5,6,7,9 (eigenvalue $=1,096$ ). The factor load of each individual SAVE-9 scale item was greater than 0.5. Detailed data are presented in Table 6 .

The total score of SAVE-9 with a high degree of confidence predicted the GAD-7 value. The ROC graph displayed the convex pattern that indicated good discrimination ability (see Figure 1). Area Under the Curve (AUC) demonstrated solid diagnostic accuracy

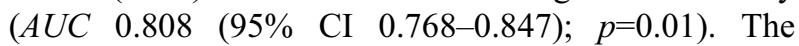
appropriate cut-off score was determined as 18 with good sensitivity and specificity $(A U C=0.808$, sensitivity $=0.68 \sim 0.73$, specificity $=0.76 \sim 0.8)$.

\section{DISCUSSION}

This study revealed that a substantial proportion of healthcare workers involved with the COVID-19 pandemic in Russia have mental health issues similarly to prior reports from China and Italy (Lui et al. 2012, Hall et al. 2008). 12.3\% of medical staff in Russia had severe anxiety compared with $19.80 \%$ in Italy and $6.2 \%$ in China according to GAD-7 total score interpretation. The median score on the GAD-7 for anxiety was 5.0 in Russia compared with 4.0 and 9.0 in China and Italy, respectively. Female gender was associated with higher levels of stress and anxiety according to both SAVE-9 and GAD-7 scales in our study. Younger age was associated with a higher anxiety level according to SAVE-9 among participants with severe anxiety, but with lower level according to GAD-7 in participants with mild, moderate and severe anxiety. In addition, physicians were associated with higher risk of anxiety according to GAD-7. Similarly, women reported more 
severe degrees of all mental health symptoms than other healthcare workers in Italy and China. Younger age was also associated with higher level of stress and anxiety in China. However, nurses had higher anxiety level in Italy compared with physicians. Women and nurses of younger age were associated with higher likelihood of developing mental health disorders during previous epidemics as well (Koh et al. 2005). Similar survey from Croatia reported that $17 \%$ of healthcare workers had moderate to extremely-severe anxiety and 10\% moderate to extremely-severe stress (Salopek-Žiha et al. 2020).

Prior viral outbreaks studies also showed that the long-term psychiatric outcomes in frontline healthcare professionals included emotional burnout, psychological distress, posttraumatic stress, prolonged adjustment disorder, psychoses and depression with suicidality (Chan \& Huak 2004, Khalid et al. 2016, Maunder et al. 2006). In 2006, randomly selected employees of a hospital were surveyed concerning their exposure to the 2003 Severe Acute Respiratory Syndrome (SARS) outbreak, and about $10 \%$ of the respondents had experienced high levels of posttraumatic stress (PTS) symptoms since the outbreak. Respondents, who worked in high-risk locations or who had friends or close relatives that contracted SARS, were 2 to 3 times more likely to have high PTS symptom levels, than those without these exposures (Wu et al. 2009). Thus, it is important to identify stress and anxiety symptoms in early stages with valid diagnostic tools in order to provide appropriate psychological support and treatment.

Another objective of our study was to validate the Russian version of new SAVE-9 scale. The internal consistency was good (Cronbachs' alpha - 0.787) and comparable with original scale. The total score of SAVE-9 with a high degree of confidence could predict the GAD-7 value. The sensitivity and specificity of SAVE-9 compared with GAD-7 was 0.808 (AUC 95\% CI $0.768-0.847 ; p=0.01)$, and an appropriate cut-off score with good sensitivity and specificity was determined as 18 , which is lower than 22 proposed by our Korean colleagues, who included the medical staff of one hospital with a necessity of psychological support (Chung et al. 2020).

The main concern of medical workers according to SAVE-9 questionnaire was their family or friends becoming infected because of them. Participants also have been worried about getting infected themselves and their health worsening because of COVID-19. Other questions contributed less to the total SAVE-9 score. As a result of our principal components analysis we confirmed the two-factor structure of our Korean colleagues and developers of SAVE-9 (Chung et al. 2020). First 'anxiety' factor included items 2,3,4,8 and second 'work-related stress' factor - items 1,5,6,7,9. So in contrast to the data of Korean validation study, questions 1 ("Are you afraid the virus outbreak will continue indefinitely?") and 5 ("Are you worried that others might avoid you even after the infection risk has been minimized?") from this study entered to the second factor with lower loading. The results may be sample and culture dependent and could explain that both items at least partly reflect not only pure anxiety but also a social stress and depression associated with long-term negative projections and possible social stigmatization. Indirectly it's confirmed by the presence of item 9 in the second factor with comparable loading, that partly indicate to negative affect and ruminations of guilt. Additional studies from different regions with larger samples and comparison against validated stress and depression scales could clarify this disagreement of SAVE-9 factor structure.

This is the first study outside Korea on validation of the new SAVE-9 scale in a large sample of medical workers involved with the COVID-19 pandemic. It's important that in comparison with other widely used self-rating screening tools for detecting stress, anxiety and depression like GPS (Olff et al. 2020), PSS (Cohen \& Hoberman 1983), IADQ (Young 1998), GAD-7 (Spitzer et al. 1999), PHQ-9 (Spitzer et al. 2006), BDI (Steer et al. 1986), the new SAVE-9 scale was elaborated to evaluate the psychological status of healthcare workers specifically in response to viral outbreaks. These types of new scales, for example, Coronavirus Anxiety Scale have already demonstrated solid reliability and validity on the general population (Lee 2020). Using such mental health screeners to identify healthcare personnel with stress and anxiety symptoms and providing them with mental health support and treatment is an important step toward combating the COVID-19 pandemic.

Unfortunately, nowadays, many barriers limit the immediate implantation of such support programs due to the quarantine policy; however, self-help interventions (Yang et al. 2020) spread of online materials on stress and anxiety reduction, implementation of a psychological assistance hotline, and involvement in leisure activities among healthcare workers and family support may be helpful (Chen et al. 2020, Xiang et al. 2020, Jakovljevic et al. 2020).

This study has several limitations. First, it was limited in scope. Most participants lived in Moscow where working conditions might be better compared with other regions. The study was performed just past the peak of the COVID-19 epidemic in Moscow, and was carried out over 14 days without any follow-up. No comparison with other than GAD-7 scales for work-related stress, anxiety and depression validated in the Russian Federation was possible because no such validated tools exist (Neznanov et al. 2017). The bias related to anonymous online survey could not be excluded; we had to follow this design due to the pandemic situation, although faceto-face interviews would have been more accurate in assessing the SAVE-9 score. Thus, long-term psychological implications of the pandemic and future validation of the SAVE-9 with face-to-face interviews are worth further investigation. 


\section{CONCLUSIONS}

Our study has shown that healthcare workers in Russia practicing treatment of patients with COVID-19 reported high rates of stress and anxiety similar to other countries. Female gender, younger age and being a physician were associated with higher levels of anxiety. These results demonstrate the importance of supportive programs for healthcare workers fighting COVID-19.

The SAVE-9 scale showed good diagnostic accuracy for the evaluation of stress and anxiety among healthcare workers during a viral outbreak. It could therefore be recommended as a more precise diagnostic tool for identification of physicians and nurses who are more vulnerable to work-related stress associated with the coronavirus and potential future treatment of this stress.

\section{Acknowledgements:}

We are thankful to all the healthcare workers in Russian COVID-19 medical centers who voluntarily participated in our online survey

Conflict of interest: None to declare.

\section{Contribution of individual authors:}

Ekaterina Mosolova \& Sergey Mosolov designed this work, collected and interpreted the data.

Ekaterina Mosolova contributed to data analysis and interpretation and drafted the manuscript.

Seockhoon Chung contributed to the writing, study concept, data interpretation, he is the author of original SAVE-9 scale.

Dmitry Sosin interpreted the data, contributed to data analysis figures and tables.

All authors helped interpret the data and critically reviewed and revised the manuscript content and approved the final version.

\section{References}

1. Cai H, Tu B, Ma J, Chen L, Fu L, Jiang Y, et al.: Psychological Impact and Coping Strategies of Frontline Medical Staff in Hunan Between January and March 2020 During the Outbreak of Coronavirus Disease 2019 (COVID-19) in Hubei, China. Med Sci Monit 2020; 26: e924171. https://doi.org/10.12659/MSM.924171.

2. Chan AO \& Huak CY: Psychological impact of the 2003 severe acute respiratory syndrome outbreak on health care workers in a medium size regional general hospital in Singapore. Occup Med (Lond) 2004; 54:190-196. https://doi.org/10.1093/occmed/kqh027

3. Chen Q, Liang M, Li Y, Guo J, Fei D, Wang L, et al.: Mental health care for medical staff in China during the COVID-19 outbreak. The lancet. Psychiatry 2020; 7: e15e16. https://doi.org/10.1016/S2215-0366(20)30078-X

4. Chung S, Kim HJ, Ahn MA, Yeo S, Lee J, Kim K, et al.: Development of the Stress and Anxiety to Viral Epidemics9 (SAVE-9) scale for assessing work-related stress and anxiety in healthcare workers in response to COVID-19.
PsyArXiv 2020; [Preprint].

https://doi.org/10.31234/osf.io/a52b4

5. Cohen $S \&$ Hoberman HM: Positive events and social supports as buffers of life change stress. J Appl Soc Psychol 1983; 13:99-125.

https://doi.org/10.1184/R1/6617633.v1

6. Hall RC, Hall RC \& Chapman MJ: The 1995 Kikwit Ebola outbreak: lessons hospitals and physicians can apply to future viral epidemics. Gen Hosp Psychiatry 2008; 30:446-452. https://doi.org/10.1016/j.genhosppsych.2008.05.003

7. Ho CS, Chee CY \& Ho RC: Mental Health Strategies to Combat the Psychological Impact of COVID-19 Beyond Paranoia and Panic. Ann Acad Med Singapore 2020; 49:155-160.

8. Jakovljevic $M$, Bjedov $S$, Jaksic $N$, \& Jakovljevic I: COVID-19 Pandemia and Public and Global Mental Health from the Perspective of Global Health Securit. Psychiatr Danub 2020; 32:6-14. https://doi.org/10.24869/psyd.2020.6

9. Khalid I, Khalid TJ, Qabajah MR, Barnard AG \& Qushmaq IA: Healthcare Workers Emotions, Perceived Stressors and Coping Strategies During a MERS-CoV Outbreak. Clin Med Res 2016;14:7-14. https://doi.org/10.3121/cmr.2016.1303.

10. Koh D, Lim MK, Chia SE, Ko SM, Qian F, Ng V, et al.: Risk perception and impact of Severe Acute Respiratory Syndrome (SARS) on work and personal lives of healthcare workers in Singapore: what can we learn?. Med Care 2005;43: 676-682. https://doi.org/10.1097/01.mlr.0000167181.36730.cc

11. Lai J, Ma S, Wang Y, Cai Z, Hu J, Wei N, et al.: Factors Associated With Mental Health Outcomes Among Health Care Workers Exposed to Coronavirus Disease 2019. JAMA Netw Open 2020; 3:e203976. https://doi.org/10.1001/jamanetworkopen.2020.3976

12. Lee SA: Coronavirus Anxiety Scale: A brief mental health screener for COVID-19 related anxiety. Death Studies 2020; 44:393-401. https://doi.org/10.1080/07481187.2020.1748481

13. Liu X, Kakade M, Fuller CJ, Fan B, Fang Y, Kong J, et al.: Depression after exposure to stressful events: lessons learned from the severe acute respiratory syndrome epidemic. Compr Psychiatry 2012; 53:15-23. https://doi.org/10.1016/j.comppsych.2011.02.003

14. Löwe B, Decker O, Müller S, Brähler E, Schellberg D, Herzog $W$, et al.: Validation and standardization of the Generalized Anxiety Disorder Screener (GAD-7) in the general population. Med Care 2008; 46:266-74. https://doi.org/10.1097/MLR.0b013e318160d093.

15. Lung FW, Lu YC, Chang YY \& Shu BC: Mental Symptoms in Different Health Professionals During the SARS Attack: A Follow-up Study. Psychiatr $Q$ 2009; 80:107-116. https://doi.org/10.1007/s11126-009-9095-5

16. Maunder RG, Lancee WJ, Balderson KE, Bennett JP, Borgundvaag B, Evans $S$ et al.: Long-term psychological and occupational effects of providing hospital healthcare during SARS outbreak. Emerg Infect Dis 2006; 12: 19241932. https://doi.org/10.3201/eid1212.060584

17. Mohindra $R, R R$, Suri V, Bhalla A \& Singh SM: Issues relevant to mental health promotion in frontline health care providers managing quarantined/isolated COVID19 patients: Asian J Psychiatr 2020; 51: 102084. https://doi.org/10.1016/j.ajp.2020.102084 
18. Незнанов НГ, Мартынихин ИА, Мосолов СН: Диагностика и терапия тревожных расстроиств в Россиискои Федерации: результать опроса врачеи психиатров. Современная терапия психических paccтройств 2017; 2:2-15. [Neznanov NG, Martynihin IA \& Mosolov SN. Diagnostika i terapya trevozhnyh rassrtojstv $v$ Rossijskoj Federatsii: rezultaty oprosa vrachei psyhiatrov. Sovrem. ter. psih. rasstrojstv 2017; 2:2-15 https://doi.org/10.21265/PSYPH.2017.41.6437]

19. Olff M, Bakker A, Frewen P, Aakvaag H, Ajdukovic D, Brewer D, et al.: Screening for consequences of trauma an update on the global collaboration on traumatic stress. Eur J Psychotraumatol 2020; 11:1752504. https://doi.org/10.1080/20008198.2020.1752504

20. Rossi R, Socci V, Pacitti F, Di Lorenzo G, Di Marco A, Siracusano A, et al.: Mental Health Outcomes Among Frontline and Second-Line Health Care Workers During the Coronavirus Disease 2019 (COVID-19) Pandemic in Italy. JAMA Netw Open 2020; 3:e2010185. https://doi.org/10.1001/jamanetworkopen.2020.10185

21. Salopek-Žiha D, Hlavati M, Gvozdanović Z, Gašić $M$, Placento H, Jakić $H$ et al.: Differences in Distress and Coping with the COVID-19 Stressor in Nurses and Physicians. Psychiatr Danub 2020; 32:287-293. https://doi.org/10.24869/psyd.2020.287

22. Sohrabi C, Alsafi Z, O'Neill N, Khan M, Kerwan A, AlJabir A, et al.: Corrigendum to "World Health Organization declares Global Emergency: A review of the 2019 Novel Coronavirus (COVID-19)" Int J Surg 2020; 77:217. https://doi.org/10.1016/j.ijsu.2020.03.036

23. Spitzer RL, Kroenke $K \&$ Williams JB: Validation and utility of a self-report version of PRIME-MD: the PHQ primary care study. Primary Care Evaluation of Mental Disorders. Patient Health Questionnaire. JAMA 1999; 282:1737-1744.

https://doi.org/10.1001/jama.282.18.1737
24. Spitzer RL, Kroenke K, Williams JB \& Löwe B: A brief measure for assessing generalized anxiety disorder: the GAD-7. Arch Intern Med 2006; 166: 1092-1097. https://doi.org/10.1001/archinte.166.10.1092

25. Steer RA, Beck AT \& Garrison B: Applications of the Beck Depression Inventory. In: Sartorius $N$, Ban TA, eds. Assessment of Depression. Geneva, Switzerland: World Health Organization, 1986; 121-142. https://doi.org/10.1023/A:1020601806080

26. World Health Organization: WHO Virtual press conference on COVID-19, https://www.who.int/docs/defaultsource/coronaviruse/transcripts/who-audio-emergenciescoronavirus-press-conference-full-and-final 11 mar2020. pdf?sfvrsn=cb432bb3_2 [accessed June 1, 2020]

27. Wu P, Fang Y, Guan Z, Fan B, Kong J, Yao Z, et al.: The psychological impact of the SARS epidemic on hospital employees in China: exposure, risk perception, and altruistic acceptance of risk. Can J Psychiatry 2009; 54: 302-11. https://doi.org/10.1177/070674370905400504

28. Xiang YT, Yang Y, Li W, Zhang L, Zhang Q, Cheung T, et al.: Timely mental health care for the 2019 novel coronavirus outbreak is urgently needed. Lancet Psychiatry 2020; 7:228-29. https://doi.org/10.1016/S2215-0366(20)30046-8

29. Xiao H, Zhang Y, Kong D, Li S \& Yang N: The Effects of Social Support on Sleep Quality of Medical Staff Treating Patients with Coronavirus Disease 2019 (COVID-19) in January and February 2020 in China. Med Sci Monit 2020; 26: e923549. https://doi.org/10.12659/MSM.923549

30. Yang L, Yin J, Wang D, Rahman A \& Li X: Urgent need to develop evidence-based self-help interventions for mental health of healthcare workers in COVID-19 pandemic. Psychol Med 2020; 1-2. https://doi.org/10.1017/S0033291720001385

31. Young KS: Internet addiction: the emergence of a new clinical disorder. Cyberpsychol Behav 1998; 1:237-44

Correspondence:

Sergey Mosolov, MD

Moscow Research Institute of Psychiatry

3 Poteshnaya ul., 107076 Moscow, Russia

E-mail:profmosolov@mail.ru 